\title{
Estrés por aluminio en plantas: reacciones en el suelo, síntomas en vegetales y posibilidades de corrección. Una revisión
}

\section{Stress for aluminum in plants: reactions in the soil, symptoms in plants and amelioration possibilities. A review}

FANOR CASIERRA-POSADA ${ }^{1}$

OSCAR E. AGUILAR-AVENDAÑ̃²

Horizontes constituyentes del perfil de un suelo ácido en Boyacá.

Foto: F. Casierra-Posada

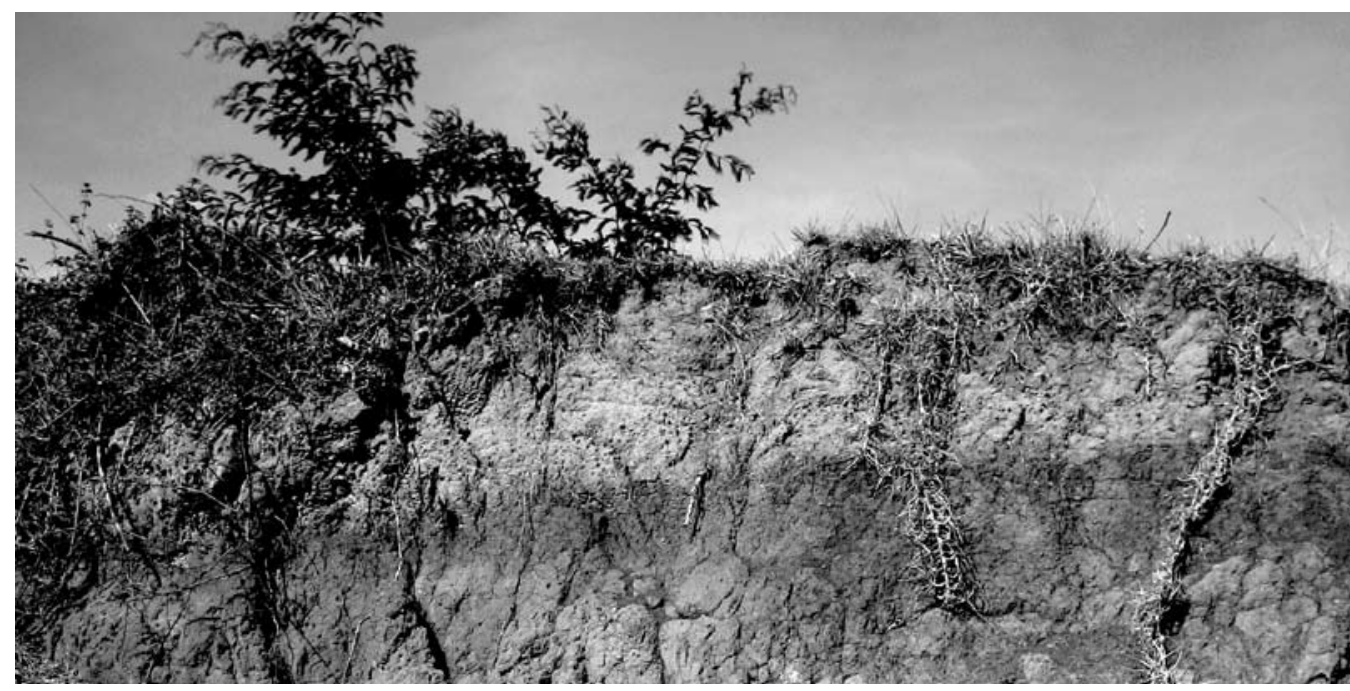

\section{RESUMEN}

El aluminio es el factor más limitante del crecimiento y productividad en los suelos ácidos del mundo, que abarcan más de $40 \%$ de la superficie agrícola. Alrededor de $85 \%$ del territorio colombiano está compuesto por suelos ácidos, en los cuales la productividad de plantas se restringe debido a la acidez del suelo y la toxicidad por aluminio. En suelos minerales ácidos con pH inferior a 5,5 gran proporción de los sitios de intercambio

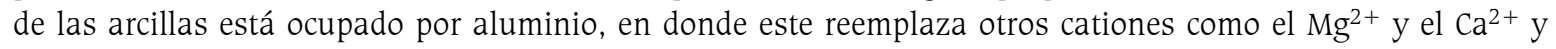
simultáneamente se adsorbe a los fosfatos. El efecto de competencia del aluminio sobre la toma de calcio y magnesio justifica que las relaciones $\mathrm{Ca} / \mathrm{Al}, \mathrm{Mg} / \mathrm{Al} \circ(\mathrm{Ca}+\mathrm{Mg}+\mathrm{K}) / \mathrm{Al}$ en el suelo o en las soluciones nutritivas son mejores parámetros para predecir el riesgo de deficiencia de bases inducidas por aluminio, que la concentración de alguno de los elementos en forma individual. El efecto inicial del estrés por aluminio es la inhibición del crecimiento radicular, además, la acumulación de aluminio indica que la sensibilidad al elemento ocurre en el ápice radicular; los mecanismos de resistencia a aluminio están confinados principalmente en el ápice de la raíz; la producción de calosa es inducida en células apicales radiculares. Algunas posibilidades de corrección

Profesor Asociado, Grupo de Investigación Ecofisiología Vegetal, Facultad de Ciencias Agropecuarias, Universidad Pedagógica y Tecnológica de Colombia, Tunja (Colombia).fanor.casierra@uptc.edu.co

2 Estudiante de Ingeniería Agronómica, Grupo de Investigación Ecofisiología Vegetal, Facultad de Ciencias Agropecuarias, Universidad Pedagógica y Tecnológica de Colombia, Tunja (Colombia).oskareduardo1@hotmail.com 
del estrés por aluminio son: la aplicación de materia orgánica, la colonización con micorrizas, la aplicación de ácidos orgánicos, la aplicación foliar de fósforo, el encalado, la selección de plantas tolerantes y la producción de plantas transgénicas tolerantes.

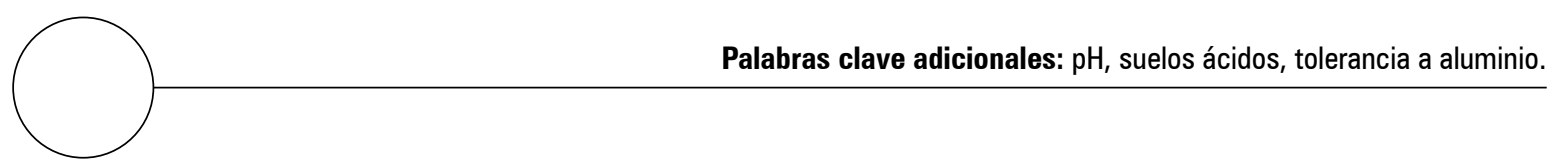

\section{ABSTRACT}

In the most acid soils worldwide, covering up to $40 \%$ of the surface potentially usable for crops, aluminum is the most growth and yield limiting factor. It is estimated that about $85 \%$ of the total land surface in Colombia is composed by acid soils where the productivity of most food crops is severely restricted due to soil acidity and aluminum toxicity. In acid mineral soils with $\mathrm{pH}$ values below 5,5 a large proportion of the cation exchange sites of clay minerals is occupied by aluminum where it specially replaces other polyvalent cations as $\mathrm{Mg}^{2+}$ and $\mathrm{Ca}^{2+}$ and simultaneously acts as a strong absorber of phosphate. The strong competing effect of aluminum on calcium and magnesium uptake explains why the molar ratios of $\mathrm{Ca} / \mathrm{Al}, \mathrm{Mg} / \mathrm{Al}$ or $(\mathrm{Ca}+\mathrm{Mg}+\mathrm{K}) / \mathrm{Al}$ in the soil or nutrient solutions are sometimes better parameters for predicting of the risk of aluminum-induced bases deficiency than the concentrations of any individual elements. The initial effect of aluminum stress is the inhibition of root growth, moreover aluminum accumulation is an indicator of Al-sensitivity takes place in the root apex; Al-resistance mechanisms are confined mainly to the root apex; and callose formation is induced in apical cells of the roots. Some amelioration possibilities to aluminum stress are: application of organic matter to the soil, root colonization with mycorrhizas, application of organic acids to the nutrient solution, foliar application of phosphorus, and lime application to the soil, Al-tolerant plants selection, and transgenic tolerant plants.

Additional key words: $\mathrm{pH}$, acid soils, Al-tolerance.

Fecha de recepción: 20-09-2007

Aprobado para publicación: 28-11-2007
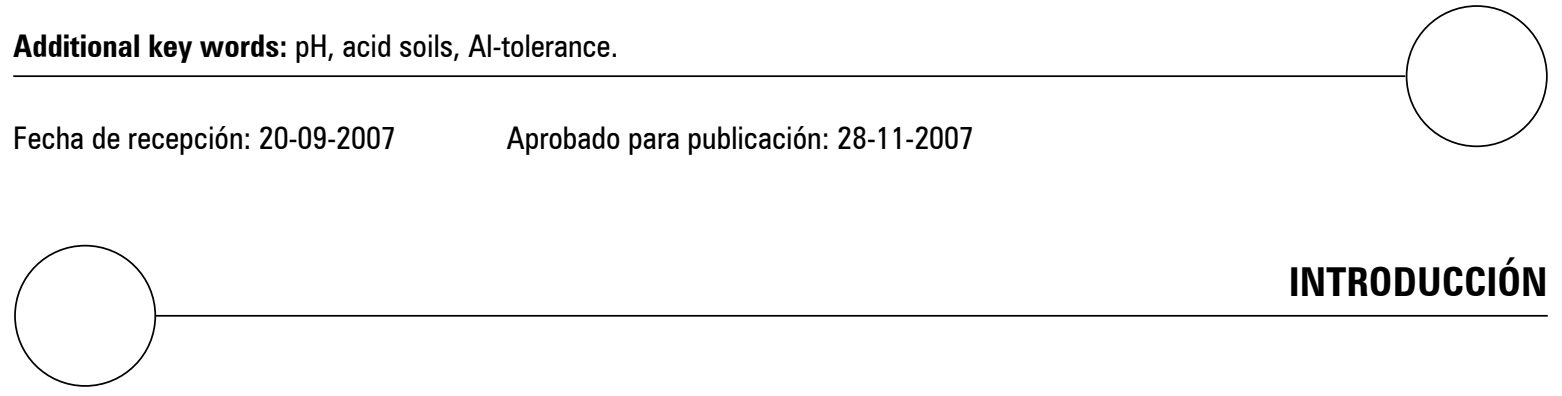

El estudio de la reacción de las plantas cultivadas en suelos ácidos reviste gran importancia para las condiciones colombianas, dado que por encima del $85 \%$ del territorio nacional presenta valores de $\mathrm{pH}$ en el suelo por debajo de 5,5 lo cual ocasiona el incremento en la concentración de $\mathrm{H}^{+}$, niveles elevados de aluminio y manganeso, la reducción de la concentración de cationes macronutrientes, así como la reducción de la solubilidad del fósforo y del molibdeno, y por último, causa inhibición del crecimiento radical, la cual tiene como consecuencia una reducción en la toma de agua y de nutrientes. Es claro que estos efectos de la reacción del suelo sobre las plantas serán más o menos acentuados, de acuerdo con las propiedades físico químicas propias del suelo (Casierra-Posada, 2001; Zapata, 2004).

A través del presente texto se discuten las reacciones del aluminio en el suelo, la interacción aluminio/raíz, y por último, se presentan algunas opciones para contrarrestar la toxicidad por alu- 
minio en las plantas. Algunas de estas alternativas no están al alcance de los agricultores, pero representan una posibilidad para el manejo futuro del estrés por aluminio en condiciones de campo.

\section{EL ALUMINIO EN EL SUELO}

Por encima de $15 \%$ de la corteza terrestre está compuesta por $\mathrm{Al}_{2} \mathrm{O}_{3}$, el cual es tan poco soluble en suelos neutros o alcalinos que no alcanzaría concentraciones tóxicas para los vegetales; sin embargo, con la reducción del pH del suelo se incrementa la solubilidad del aluminio hasta llegar a ocupar más de la mitad de los sitios de intercambio iónico del suelo (Wallnöfer y Engelhardt, 1995). La concentración de $\mathrm{Al}^{3+}$ en la solución del suelo a pH menor de 5,0 se encuentra en el rango de 10 - $100 \mu \mathrm{M}$, e incluso, en suelos minerales de ecosistemas forestales, la concentración de $\mathrm{Al}^{3+}$ soluble puede alcanzar valores cercanos a $1.000 \mu \mathrm{M}$; el aluminio se reporta como el factor más limitante de la producción agrícola en suelos ácidos, los cuales comprenden alrededor del $40 \%$ de la superficie cultivable en el mundo (Matzner y Prenzel, 1992; Ma, 2000).

Con la reducción del pH del suelo, sucede la destrucción de los minerales de las arcillas y de otros silicatos, así como la solubilización de óxidos de aluminio, lo que conduce a la degradación irreversible del suelo; el aluminio aparece en estos casos como $\mathrm{Al}^{3+}$. La meteorización de los componentes del suelo permite la liberación de iones $\mathrm{Al}^{3+}$ a partir de la red de los silicatos de las arci- llas. El proceso de meteorización de los silicatos de aluminio de las arcillas tiene como resultado la aparición de iones $\mathrm{H}^{+}$, los cuales pueden tener dos posibilidades de origen: (1) provienen de las cargas negativas permanentes producidas por sustitución isomórfica de un elemento como el $\mathrm{Al}^{3+}$ por otro como el $\mathrm{Si}^{3+}$, con liberación de una carga negativa; (2) también se pueden originar a partir de la disociación de iones $\mathrm{H}^{+}$en los grupos $\mathrm{OH}^{-}$(Guerrero, 1991). Adicionalmente, los protones del suelo tienen otros orígenes. En los suelos ácidos, estas fuentes pueden ser la hidrólisis del $\mathrm{CO}_{2}$ proveniente de la respiración de los microorganismos, la hidrólisis de cationes metálicos, los grupos ácidos y alcohólicos de la materia orgánica, los grupos $\mathrm{OH}^{-}$de las láminas de los aluminosilicatos y los fertilizantes. Igualmente, al suelo pueden llegar ácidos fuertes provenientes de contaminantes como la lluvia ácida o vertidos industriales (Zapata, 2004). En la tabla 1 se puede observar que el contenido de aluminio en algunos de los minerales más frecuentes de encontrar en los suelos, es bastante alto en comparación con el contenido de calcio y magnesio, de manera que la destrucción de los componentes de las arcillas, permite la liberación de grandes cantidades de aluminio.

En horizontes del suelo ricos en materia orgánica, la mayor parte de los iones de aluminio forman complejos no tóxicos con los compuestos húmicos. Además, algunos iones de aluminio pueden incorporarse a las capas intermedias de los minerales expandidos de las arcillas y así sucede una transformación de estos minerales

Tabla 1. Rango de variación de la composición química en algunos minerales del suelo (\%), según Scheffer y Schachstabel (1995).

\begin{tabular}{|l|r|r|r|r|r|r|r|r|}
\multicolumn{1}{|c|}{ Mineral } & \multicolumn{1}{c|}{$\mathrm{SiO}_{2}$} & \multicolumn{1}{c|}{$\mathrm{Al}_{2} \mathrm{O}_{3}$} & \multicolumn{1}{c|}{$\mathrm{Fe}_{2} \mathrm{O}_{3}$} & \multicolumn{1}{c|}{$\mathrm{Ti}_{2} \mathrm{O}_{3}$} & \multicolumn{1}{c|}{$\mathrm{CaO}$} & \multicolumn{1}{c|}{$\mathrm{MgO}$} & $\mathrm{K}_{2} \mathrm{O}$ & $\mathrm{Na}_{2} \mathrm{O}$ \\
\hline Caolinita & $45-48$ & $38-40$ & $0-0,2$ & $0-0,3$ & - & - & - & - \\
\hline Smectita & $42-55$ & $0-28$ & $0-30$ & $0-0,5$ & $0-0,3$ & $0-0,5$ & $0-0,5$ & $0-3$ \\
\hline Illita & $50-56$ & $18-31$ & $2-5$ & $0-0,8$ & $0-2$ & $1-4$ & $4-7$ & $0-1$ \\
\hline Vermiculita & $33-37$ & $7-18$ & $3-12$ & $0-0,6$ & $0-2$ & $20-28$ & $0-2$ & $0-0,4$ \\
\hline Clorita & $22-35$ & $12-24$ & $0-15$ & - & $0-2$ & $12-34$ & $0-1$ & $0-1$ \\
\hline
\end{tabular}


en cloruros de aluminio, con una capacidad de intercambio catiónico considerablemente reducida. En los horizontes pobres en materia orgánica con alta concentración de sulfatos, bajo condiciones de reacción ácida del suelo, una parte de los iones $\mathrm{OH}^{-}$pueden ser reemplazados por $\mathrm{SO}_{4}{ }^{2-}$; el aluminio puede asociarse a los sulfatos y formar hidroxisulfatos de aluminio como la Jurbanita $\left(\mathrm{AlOHSO}_{4}\right)$. Por otra parte, sucede la adsorción de $\mathrm{Al}^{3+}$ e hidróxidos de aluminio, como $\mathrm{AlHO}^{2+}$, $\mathrm{Al}(\mathrm{OH})_{2}{ }^{+} \mathrm{y} \mathrm{Al}_{\mathrm{x}}(\mathrm{OH})_{\mathrm{y}}{ }^{(3 \mathrm{x}-\mathrm{y})+}$, a la superficie de los minerales de las arcillas. Como consecuencia de la intensidad del intercambio de iones de aluminio, los cationes de los elementos nutritivos son desplazados de los sitios de intercambio y lavados posteriormente. Por otro lado, algunos tipos monovalentes de aluminio asociados a compuestos inorgánicos, como $\mathrm{AlF}^{2+}, \mathrm{AlF}^{2+} \mathrm{o} \mathrm{AlSO}_{4}{ }^{+}$, no representan riesgo de fitotoxicidad para los vegetales.

En condiciones de $\mathrm{pH}$ extremadamente ácido [pH $\left(\mathrm{CaCl}_{2}\right)<3$ ] comienza la solubilización del óxido de hierro cristalino Ferrihydrita, lo que conduce a la liberación de iones $\mathrm{Fe}^{3+}$. A ese rango de $\mathrm{pH}$ casi $100 \%$ de los cationes de cambio están conformados por $\mathrm{Al}^{3+}$, y una parte muy pequeña por $\mathrm{H}^{+}, \mathrm{Fe}^{3+} \mathrm{y} \mathrm{Mn}^{2+}$. Como consecuencia, en las plantas se presentan deficiencias nutritivas severas y efectos tóxicos causados por los iones de aluminio y manganeso.

La magnitud de la toxicidad por aluminio depende del contenido de elementos nutritivos en el suelo, especialmente magnesio y calcio; por tanto, la relación $\mathrm{Ca} / \mathrm{Al}, \mathrm{Mg} / \mathrm{Al}$ y $(\mathrm{Ca}+\mathrm{Mg}+\mathrm{K}) / \mathrm{Al}$ en la solución del suelo resultan ser parámetros de gran importancia para determinar la posibilidad de toxicidad por aluminio. En esta última relación, se considera que el aluminio presenta condiciones de toxicidad para la planta, cuando el cociente es menor o igual que la unidad (Instituto Colombiano Agropecuario, 1992).

La flora y fauna del suelo también sufren las consecuencias de la toxicidad por aluminio. Es así como en un pH en el rango 3,5-4,0, se encuen- tran por lo general pocas poblaciones de anélidos en el suelo y la cantidad de micorrizas es muy reducida; además, muchos grupos de bacterias y actinomicetos reducen su actividad y desarrollo cuando el pH del suelo está por debajo de 5,5.

\section{TOMA DE ALUMINIO POR LA PLANTA}

El aluminio puede ser tomado por la planta con gran rapidez principalmente cuando el suelo tiene una reacción ácida. Las reacciones químicas del aluminio en el suelo son extraordinariamente complejas y diversas, y comprenden principalmente hidrólisis, polimerización y reemplazo de elementos. Algunos monómeros resultantes de las reacciones de hidrólisis de los compuestos de aluminio han sido reconocidos como fitotóxicos, sin embargo, los reportes sobre el efecto del hidróxido de aluminio en los vegetales son contradictorios. Las reacciones de hidrólisis del aluminio semejan la reacción de un ácido fuerte, en la cual se liberan iones protones (Wallnöfer y Engelhardt, 1995).

El $\mathrm{Al}(\mathrm{OH})_{2} \cdot 5 \mathrm{H}_{2} \mathrm{O}$ es el compuesto de aluminio que tiene el efecto más nocivo sobre el desarrollo de Triticum aestivum, mientras que $\mathrm{Al}^{3+} \cdot 6 \mathrm{H}_{2} \mathrm{O}$ y $\mathrm{Al}(\mathrm{OH})^{2+} .4 \mathrm{H}_{2} \mathrm{O}$ son los compuestos que más daño ocasionan a las plantas de Coffea arabica. El crecimiento radicular en plantas de Glycine max se reduce en presencia de $\mathrm{Al}(\mathrm{OH})^{2+} .5 \mathrm{H}_{2} \mathrm{O}, \mathrm{Al}$ $\left(\mathrm{SO}_{4}\right)^{+}, \mathrm{Al}(\mathrm{OH})^{2+} \cdot 4 \mathrm{H}_{2} \mathrm{O}$ y Al${ }^{3+} \cdot 6 \mathrm{H}_{2} \mathrm{O}$. De esta manera se puede apreciar que los polímeros de aluminio son en general más tóxicos que las formas monoméricas del elemento y sin embargo, con frecuencia se tienen como compuestos de poca importancia en casos de contaminación del suelo por aluminio (Wallnöfer y Engelhardt, 1995). Mediante espectrometría de resonancia nuclear (27Al-NMR), Kloke (1978) encontró algunas formas de $\mathrm{AlO}_{4} \mathrm{Al}_{12}(\mathrm{OH})_{24}\left(\mathrm{H}_{2} \mathrm{O}\right)_{12}{ }^{7+}$ (denominado $\mathrm{Al}_{13}$ ) en muestras de suelo de horizontes espódicos. Dado que por lo menos $30 \%$ de esos polímeros de aluminio fueron determinados como solubles en agua, los polímeros derivados del aluminio 
contribuyen considerablemente a causar efectos tóxicos a los vegetales, por lo menos en el tipo de suelo analizado.

Es posible que la membrana plasmática, por su composición química, sea poco permeable a las formas ionizadas del aluminio, mientras que los compuestos de aluminio electrónicamente neutros o los complejos de aluminio, como $\mathrm{Al}(\mathrm{OH})_{3} \cdot 3 \mathrm{H}_{2} \mathrm{O}$ o $\mathrm{AlCl}_{3}$ sean más móviles. Además, el aluminio puede atravesar la membrana plasmática a través de los poros hidrófilos o por los canales de proteína y alcanzar el interior de la célula, (Wallnöfer y Engelhardt, 1995; CasierraPosada, 2001; 2002).

Es muy complicado establecer las formas individuales de aluminio que son tóxicas a los vegetales, dado que los compuestos del elemento reaccionan constantemente entre sí; por tanto, la sumatoria de las formas monoméricas de aluminio presentes en el sustrato o en la solución del suelo representan una buena alternativa para calcular el efecto probable del aluminio en las plantas.

La membrana plasmática, y de igual manera el apoplasto de las células radiculares representan una barrera para la toma del aluminio, sin embargo, puede suceder una acumulación pasiva del elemento en el apoplasto; además, es posible que el apoplasto no sea completamente permeable al aluminio, pues el tejido fuera de la endodermis presenta usualmente diferentes modelos de distribución del elemento (Casierra-Posada, 2001; 2002).

En la actualidad se acepta que el ápice radicular juega un papel muy importante en cuanto a la respuesta a la toxicidad por aluminio y a la percepción de la presencia del elemento en forma soluble en el sustrato. Con base en las investigaciones realizadas al respecto, Sivaguru et al. (2000) sintetiza en tres aspectos el papel del ápice radicular en la respuesta al estrés por aluminio: (1) La acumulación de aluminio es un indicador de que la sensibilidad al elemento se ubica en el ápice radicular. (2) Los mecanismos de tolerancia a aluminio, como la exudación de ácidos orgánicos para formar complejos con el elemento, están confinados principalmente al ápice radicular. (3) La formación de calosa como indicador de la sensibilidad a aluminio, se induce en primer lugar en las células apicales de la corteza externa de la raíz.

\section{SÍNTOMAS DE ESTRÉS POR ALUMINIO}

La concentración de $\mathrm{Al}^{3+}$ en las plantas cultivadas usualmente es más alta en las raíces que en los tejidos aéreos. El primer síntoma perceptible cuando se presenta toxicidad por $\mathrm{Al}^{3+}$ es la reducción del crecimiento radicular en longitud, efecto que en plantas de Triticum aestivum empieza una hora después de la exposición de las raíces a $\mathrm{Al}^{3+}$, asociado con un incremento del diámetro de los ápices radiculares; las raíces laterales resultan seriamente afectadas y de esta manera se disminuye considerablemente el volumen de la raíz (Ownby y Popham, 1989; Ryan et al., 1992). Las raíces afectadas por $\mathrm{Al}^{3+}$ exploran un volumen muy limitado del suelo y así se reduce su capacidad para tomar los nutrientes y el agua. $\mathrm{El} \mathrm{Al}^{3+}$ interfiere con la toma, el transporte y la utilización de los elementos nutritivos (Wright, 1989). Plantas de Brassica oleracea que se expusieron a una solución nutritiva con aluminio, no mostraron una sintomatología clara en las hojas como consecuencia de la toxicidad por el elemento; sin embargo, en las raíces, los síntomas del efecto negativo del $\mathrm{Al}^{3+}$ fueron bastante evidentes (figura 1). Es así como mientras las plantas control presentaban raíces filamentosas, largas y delgadas (planta de la izquierda), en las plantas expuestas al metal, las raíces se hacían más gruesas y cortas en la medida que la relación $(\mathrm{Ca}+\mathrm{Mg}+\mathrm{K}) / \mathrm{Al}$ de la solución nutritiva tenía un valor más reducido (planta a la derecha).

Los efectos de la toxicidad por $\mathrm{Al}^{3+}$ son más evidentes en los ápices radiculares, posiblemente debido a que su permeabilidad celular es mayor que en otras zonas de la raíz. Wagatsuma et al. (1987) demostraron que en Zea mays y Pisum 


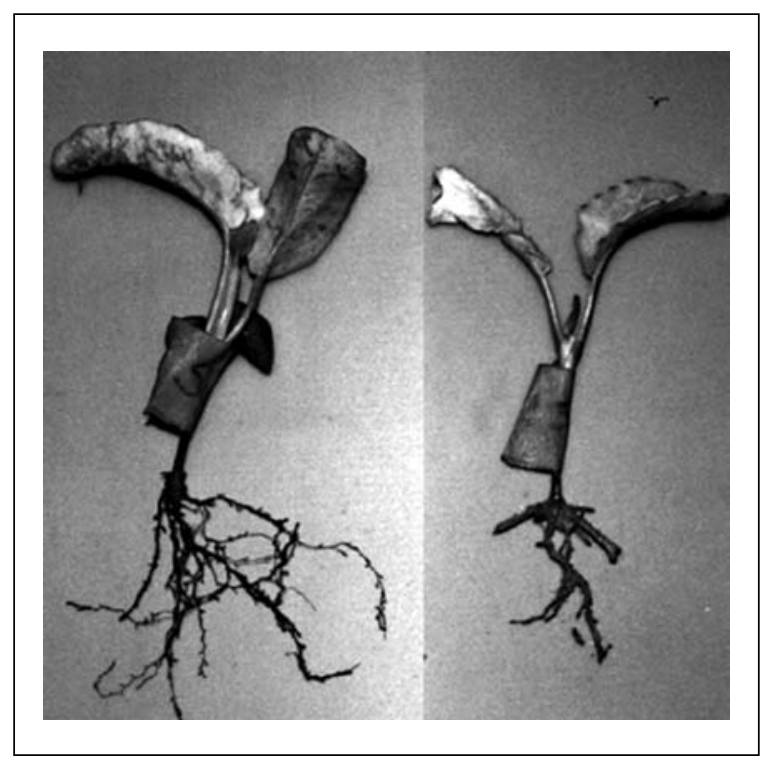

Figura 1. Apariencia externa de las raíces de plantas de coliflor (Brassica oleracea var. Botrytis) expuestas (derecha) o no (izquierda) a aluminio en la solución nutritiva (CasierraPosada y Cárdenas-Hernández, 2007). sativum la destrucción de las células corticales y epidérmicas de los ápices radiculares sucede después de 48 h de exposición a $\mathrm{Al}^{3+}$. De Lima y Copeland (1994) observaron que en Triticum aestivum, la exposición a $\mathrm{Al}^{3+}$ provoca la formación de depresiones en la epidermis de los ápices radiculares, la formación de vacuolas en esas células se incrementa, se reduce su turgencia y se producen desórdenes citoplasmáticos en las células de la epidermis y de la periferia de los meristemos radiculares. Aponte y Valencia (1983) observaron que la exposición de Coffea arabica a aluminio inducía crecimiento limitado de la parte aérea y ocurrencia de raíces gruesas, cortas y carentes de raicillas. A nivel microscópico observaron daños en los tejidos radicales, entre los cuales fueron notorias las alteraciones en la morfología y los indicios de alteración o inhibición de la división celular. Pintro et al. (1995) encontraron que la exposición a $\mathrm{Al}^{3+}$ en Zea mays provocó una reducción significativa en el crecimiento radicular y en la producción de biomasa en las raíces, lo que explican mediante un incremento del diá- metro de las raíces o mediante un aumento de la densidad celular en las mismas.

La inhibición del crecimiento y el engrosamiento de raíces expuestas a aluminio sugieren que el citoesqueleto puede ser el blanco de la toxicidad por el metal. Mediante microscopia indirecta de inmunofluorescencia, se visualizaron los microtúbulos y los microfilamentos en raíces de Zea mays y los cambios en su organización y estabilidad se correlacionaron con los síntomas de la toxicidad por $\mathrm{Al}^{3+}$. Los estudios del crecimiento demostraron que el sitio de la toxicidad por $\mathrm{Al}^{3+}$ fue asociado a la zona de elongación. Dentro de esta región, el $\mathrm{Al}^{3+}$ dio lugar a una reorganización de los microtúbulos en la corteza interna. Sin embargo, la orientación de los microtúbulos en la corteza y en la epidermis externa no cambió, incluso después de que los síntomas crónicos de la toxicidad se manifestaran. La reorientación inducida por auxinas y la depolimerización inducida por frío en los microtúbulos de la corteza externa fueron bloqueados por el tratamiento previo con $\mathrm{Al}^{3+}$. Estos resultados sugieren que el $\mathrm{Al}^{3+}$ aumenta la estabilidad de los microtúbulos en estas células. El efecto de la estabilización de la corteza externa causada por el metal, coincidió con la inhibición del crecimiento. Los microfilamentos reorientados también fueron observados en raíces tratadas con $\mathrm{Al}^{3+}$. Estos datos demuestran que la reorganización y la estabilización del citoesqueleto están asociadas íntimamente a la toxicidad por $\mathrm{Al}^{3+}$ en raíces del maíz (Blancaflor et al., 1998).

\section{CORRECCIÓN DE LA TOXICIDAD POR ALUMINIO}

Se han generalizado tres criterios para considerar al aluminio como un problema en el suelo: (1) cuando se presentan en el suelo, valores superiores a 2 meq de aluminio intercambiable; (2) cuando el porcentaje de saturación de aluminio, en relación con los cationes intercambiables, es mayor que 25; (3) si la relación $(\mathrm{Ca}+\mathrm{Mg}+\mathrm{K}) / \mathrm{Al}$ es 
menor o igual que uno. En cualquiera de estos casos es necesaria la implementación de algún correctivo con el fin de reducir el efecto tóxico del aluminio para las plantas (Instituto Colombiano Agropecuario, 1992).

La adición de materia orgánica puede reducir la fitotoxicidad por aluminio mediante la formación de complejos entre los compuestos orgánicos con el elemento (Hue et al., 1986; Kerven et al., 1989). Los ácidos orgánicos son los responsables de la formación de los complejos con el aluminio, pero los diferentes aniones orgánicos difieren ampliamente en su capacidad para detoxificar del $\mathrm{Al}^{3+}$, los suelos y la solución del suelo. El citrato, oxalato, tartarato y el EDTA son muy efectivos en este caso, mientras que el succinato y el lactato presentan una efectividad menor o nula, en comparación con los anteriores (Bartlett y Riego, 1972; Hue et al., 1986). Adams y Moore (1983) encontraron una reducción del crecimiento radicular en plantas de Glycine max desarrolladas en subsuelo con bajo contenido de materia orgánica y una concentración de aluminio de $4 \mu \mathrm{M}$, comparadas con plantas que crecieron en el horizonte superficial del suelo, rico en materia orgánica y con una concentración de aluminio de 9-134 $\mu \mathrm{M}$. La aplicación de mulch (Duong y Diep, 1986) y abonos verdes (Hue y Amien, 1989) presentan también una gran efectividad en la reducción de los efectos tóxicos del aluminio en suelos ácidos. El ácido fúlvico es uno de los compuestos que con mayor efectividad reduce la fitotoxicidad del aluminio, por su capacidad para formar complejos con monómeros y polímeros del elemento ( $\mathrm{Su}$ thipradit et al., 1990).

Muchos ácidos orgánicos segregados por las raíces de los vegetales están implicados en la reducción de la toxicidad por aluminio y en la tolerancia de las plantas al elemento (Ma, 2000). Hue et al. (1986) encontraron que la capacidad de los ácidos orgánicos en la detoxificación del aluminio depende de la orientación de sus grupos $\mathrm{OH} /$ $\mathrm{COOH}$ y de su correspondiente posibilidad para formar una estructura estable con el elemento.
La efectividad de algunos aniones orgánicos adicionados a soluciones nutritivas ( $\mathrm{pH} 4,25)$ con el propósito de contrarrestar los efectos tóxicos del $\mathrm{Al}^{3+}$ en concentración de $500 \mu \mathrm{M}$, en plantas de Glycine max, fue investigado por Ginting et al. (1998). Se utilizaron citrato, oxalato, tartarato, malato, malonato y lactato en concentración de $1.000 \mu \mathrm{M}$ y ácido húmico $1 \mathrm{~g} \cdot \mathrm{L}^{-1}$. El grado de respuesta de los aniones orgánicos, medido en términos de cambios en la longitud radicular, fue en el siguiente orden: oxalato $>$ citrato $\approx$ tartarato $\approx$ malato $>$ malonato $>>$ lactato; el ácido húmico tuvo una efectividad similar a la que presentó el citrato. La efectividad que presentó el oxalato en la reducción de la toxicidad por aluminio, en términos de longitud radicular, se debe a que este compuesto tiene la capacidad de formar complejos 3:1 oxalato-Al, que presentan una gran estabilidad, y por lo tanto, una gran efectividad para reducir la fitotoxicidad del aluminio (Ma, 2000).

Muchos autores han estudiado la influencia del aluminio sobre la absorción, transporte y utilización del fósforo en los vegetales (Marschner, 1995). En plantas sensibles a aluminio se encontró, mediante estudios anatómicos y con microanálisis de rayos $\mathrm{x}$, precipitados de complejos de aluminio y fósforo en los espacios libres entre las células de la raíz, lo cual trae como consecuencia, una disminución en la disponibilidad del fósforo para los procesos metabólicos dependientes de este elemento. En un ensayo realizado por Calbo y Cambraia (1980) se evaluaron dos cultivares de Sorghum bicolor según su tolerancia a aluminio y se encontró una mayor concentración tanto de fósforo como de aluminio en la raíz del cultivar sensible, lo que sugiere una mayor precipitación de fósforo y de aluminio en ese cultivar, en comparación con el cultivar tolerante.

Méndez y Ribeiro (1990) evaluaron la respuesta de plantas de Lycopersicon esculentum cultivadas en soluciones nutritivas que contenían $0,2,4$ y 6 ppm de $\mathrm{Al}^{3+}$, a aplicaciones de fósforo vía foliar o adicionado a la solución nutritiva. Las 
pulverizaciones con fósforo se realizaron cuatro veces al día con una solución $4 \mathrm{M}$ de $\mathrm{NH}_{4} \mathrm{H}_{2} \mathrm{PO}_{4}$; para los tratamientos de fósforo adicionado a la solución nutritiva se utilizó la solución de Clark, (Clark, 1975), citado por los autores. Las respuestas al estrés por la presencia de $\mathrm{Al}^{3+}$, expresado en términos de reducción de la producción de biomasa, fue más marcada cuando el fósforo fue suministrado a las plantas mediante la solución nutritiva, que cuando se aplicó vía foliar; por tanto, la aplicación de fósforo vía foliar indujo una reducción de los efectos de la toxicidad por $\mathrm{Al}^{3+}$, lo cual se debió posiblemente a que la aplicación del fósforo mediante este método, previno la precipitación de fosfato de aluminio en los espacios libres aparentes del sistema radicular de las plantas de L. esculentum. En las plantas que recibieron el fósforo adicionado a la solución nutritiva debió presentarse una gran precipitación del fosfato de $\mathrm{Al}$ en los espacios libres aparentes de las raíces, lo cual causaría evidentemente mayores daños al metabolismo del fósforo en términos de reducir su disponibilidad para que sea absorbido, transportado y asimilado.

Se ha encontrado que la aplicación exógena de nitroprusido de sodio (sodium nitroprusside SNP) reduce el efecto negativo causado por $\mathrm{Al}^{3+}$ en Hibiscus moscheutos (Qiu-Ying et al., 2007). El SNP actúa como donante de óxido nítrico (NO), el cual es un mensajero de gran importancia en muchos procesos fisiológicos en plantas. Como complemento, los compuestos que destruyen el NO o que inhiben la acción de la NO-sintetasa o la nitrato-reductasa, tienen un efecto inhibidor de la elongación de la raíz, similar al que causa el $\mathrm{Al}^{3+}$; por tanto, la reducción de la inhibición de la elongación celular se correlacionó con la concentración endógena de NO, como consecuencia de la aplicación de SNP.

Mediante la aplicación de cal al suelo, es posible lograr la reducción de los efectos tóxicos del aluminio, dado que los carbonatos, óxidos, hidróxidos y silicatos de calcio o magnesio neutralizan los iones $\mathrm{H}^{+}$en la solución del suelo, a través de los iones $\mathrm{OH}^{-}$, cuando la cal reacciona con el agua del suelo $\left(\mathrm{CaCO}_{3}+\mathrm{H}_{2} \mathrm{O} \rightarrow \mathrm{Ca}+\mathrm{HCO}_{3}{ }^{-}+\right.$ $\mathrm{OH}$ ). La cal incrementa el pH del suelo al convertir el exceso de $\mathrm{H}^{+}$en $\mathrm{H}_{2} \mathrm{O}$. Además, el incremento del pH del suelo induce la precipitación del aluminio, como $\mathrm{Al}(\mathrm{OH})_{3}$, que es un compuesto insoluble (Cumming y Weinstein, 1990).

El encalado de los suelos representa una opción relativamente económica y al alcance de los agricultores colombianos que afrontan el problema del aluminio en sus suelos, pues los materiales de encalado son de fácil acceso y se adquieren a un costo relativamente bajo.

La colonización con micorrizas es un componente importante en la adaptación de las plantas a los suelos minerales ácidos con baja disponibilidad de fósforo y concentración alta de aluminio intercambiable. En plantas de Pinus rigida inoculadas con micorrizas se encontraron niveles altos de fósforo y bajos de aluminio en las hojas, en comparación con plantas no micorrizadas (Cumming y Weinstein, 1990), además, a una concentración de $200 \mu \mathrm{M}$ de aluminio en la solución nutritiva, la inhibición del crecimiento de las plántulas fue severa en plantas no micorrizadas, pero la inhibición del crecimiento se redujo notoriamente con la inoculación de micorrizas.

Algunos hongos saprófitos tienen la capacidad de tomar grandes cantidades de metales pesados y formar compuestos en sus micelios, con lo que contribuyen a reducir la toma de estos metales por parte de las plantas (Tobin et al., 1990; Colpaert y Van Assche, 1992; Berthelin et al., 1993). La presencia de $\mathrm{Al}^{3+}$ en la solución del suelo reduce considerablemente el desarrollo de las micorrizas, las que mediante el incremento en la toma del fósforo, entre otros, ayudarían a reducir los efectos tóxicos del $\mathrm{Al}^{3+}$ para los vegetales. Marschner et al. (1999) realizaron varios ensayos para evaluar la tolerancia a $\mathrm{Al}^{3+}$ en varios grupos de ectomicorrizas de los géneros Paxillus involutus y Pisolithus tinctorius, los cuales presentaron diferentes grados de tolerancia a $\mathrm{Al}^{3+}$, pero algunos 
de los grupos de micorrizas evaluados, presentaron una tolerancia a $\mathrm{Al}^{3+}$ menor de la que había sido reportada con anterioridad.

La selección y multiplicación de plantas que tengan una tolerancia a aluminio representa una alternativa interesante para contrarrestar los problemas que este elemento ocasiona a los vegetales. Las diferentes especies y los genotipos dentro de una misma especie presentan variaciones genéticas de consideración en relación con su tolerancia a aluminio. Para realizar una selección efectiva de plantas por su tolerancia a aluminio es necesaria la utilización de parámetros adecuados para la determinación de las características relacionadas con la tolerancia al elemento. A raíz de que la mayoría de los desórdenes causados por iones metálicos tienen lugar a nivel celular, las técnicas de cultivo in vitro complementadas con los ensayos de campo ofrecen una gran cantidad de posibilidades para el estudio de los mecanismos de tolerancia y para la realización de una selección de plantas. Al utilizar la técnica in vitro es necesario considerar la expresión de la toxicidad por aluminio como un fenómeno celular independiente del explante. Sin embargo, algunas metodologías pueden considerarse como representativas en estudios de selección, sólo cuando existe una respuesta muy similar entre los resultados de los ensayos de campo y los de los experimentos in vitro (Oettler et al., 1997).

Como método rápido y no destructivo para la determinación de la tolerancia a aluminio, Aniol (1991) utilizó la prueba de germinación de semillas en soluciones nutritivas que contenían diferentes concentraciones del elemento. Junto al amplio espectro de composiciones químicas de las soluciones nutritivas, rangos de $\mathrm{pH}$ y concentraciones de aluminio, se pueden utilizar la reducción en el crecimiento radicular (Cambraia et al., 1991), el contenido de aluminio en la planta (Foy, 1988), la formación de calosa en los ápices radiculares (Oettler et al., 1997; Wissemeier et al., 1998 y Collet et al.., 2000), entre otros, como parámetros para evaluar el efecto del aluminio en las plantas. Baier et al. (1996) estudiaron la influencia del aluminio sobre el crecimiento radicular de plantas de Triticosecale y utilizaron la longitud de la raíz principal como parámetro para calificar la tolerancia a aluminio, al igual que Cambraia et al. (1991) lo hicieron en diferentes cultivares de Sorghum bicolor. Estos últimos evaluaron también la longitud total de las raíces y la longitud promedio del sistema radicular como parámetros de selección a aluminio, pero la longitud de la raíz principal resultó ser el parámetro más confiable para discriminar las plantas de S. bicolor por su tolerancia a $\mathrm{Al}^{3+}$. Aniol (1996) caracterizó algunos genotipos de Triticosecale según un tratamiento de choque con aluminio a $\mathrm{pH}$ 4,5 y según la tinción de las raíces con Eriochrome cyanine R. Como parámetro de evaluación utilizaron la dimensión de la renovación de las raíces, después de una fase de 48 h sin tratamiento.

La ingeniería genética ofrece también perspectivas promisorias en cuanto a la inducción de cierta tolerancia a aluminio en vegetales. Es así como algunos genes que inducen tolerancia a aluminio se han estudiado en Arabidopsis sp., Triticum aestivum y en la levadura Saccharomyces cerevisiae (Kochian, 1995; Ezaki et al., 2000). Larsen et al. (1997) reportaron algunos mutantes de Arabidopsis resistentes (Alr) y sensibles (Als) a aluminio. Los caracteres mutantes de Alr son semidominantes y están dirigidos por dos loci independientes, pero en el caso de los mutantes Als, se presenta una gran complejidad en cuanto a los loci que dirigen las características asociadas con la sensibilidad a aluminio en Arabidopsis. Estos mutantes se obtuvieron mediante mutagénesis química con etil-metanosulfonato.

En plantas de Sorghum bicolor se investigó su tolerancia a $\mathrm{Al}^{3+}$ teniendo como parámetro de referencia el gen $A l t_{S B}$ y se encontraron múltiples alelos en el locus $A t_{S B}$ relacionados con la tolerancia al metal (Caniato et al., 2007), los cuales modulan un rango amplio de niveles de tolerancia a $\mathrm{Al}^{3+}$. También se observó segregación trans- 
gresiva en una gran cantidad de líneas de sorgo, lo que indica la existencia potencial de efectos codominantes $\mathrm{o}$ aditivos en diferentes loci con tolerancia a $\mathrm{Al}^{3+}$.

En la actualidad es posible obtener plantas transgénicas con una tolerancia elevada a aluminio, en especies de importancia económica como Nicotiana tabacum y Carica papaya, mediante la sobreexpresión del gen que dirige la producción de citrato-sintetasa (CS) a partir de Pseudomonas aeruginosa (De la Fuente et al., 1997). Mediante este procedimiento, las plantas transgénicas tenían la capacidad de producir cinco a seis veces más ácido cítrico que los testigos no modificados; además, las plantas modificadas genéticamente presentaban una resistencia a aluminio diez veces mayor que los controles. Este ejemplo nos da una idea de la magnitud de las ventajas que ofrece la ingeniería genética como herramienta biotecnológica para inducir tolerancia a aluminio en especies de importancia económica.

\section{CONCLUSIONES}

De acuerdo con la información consignada en el presente texto, se pueden concluir los siguientes aspectos:

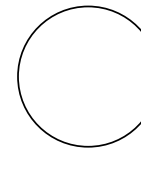

Adams, F. y B.L. Moore. 1983. Chemical factors affecting root growth in subsoil horizons of coastal plain soils. Soil Sci. Soc. Amer. J. 47, 99-102.

Aniol, A. 1991. Genetics of acid tolerant plant. pp. 10071017. En: Wright, R.J.; C. Baliger y R.P. Murrmann (eds.) Plant-Soil interactions at low pH. Kluwer Acad. Publ., Dordrecht.

Aniol, A. 1996. The variability of aluminum tolerance among triticale strains and cultivars bred in poland. pp. 461-465 En: Guedes-Pinto, P.; N. Darvey y V.P. Carnide (eds.). Triticale: Today and tomorrow. Kluwer Acad. Publ., Dordrecht.
- La sintomatología que exhiben las plantas afectadas por estrés por aluminio, no es del todo clara y puede manifestarse como la deficiencia de alguno o varios elementos nutritivos, con los cuales interactúa el aluminio.

- La adición de materiales orgánicos al suelo es de gran ayuda en la corrección de la toxicidad por aluminio, por su capacidad para formar complejos estables aluminio-orgánicos que reducen la fitotoxicidad del elemento. Esta práctica agrícola, complementada con la inoculación de las plantas con micorrizas y el encalado del suelo, reduce sustancialmente la posibilidad de estrés por aluminio para los vegetales.

- Para seleccionar materiales vegetales tolerantes a aluminio es necesario complementar las técnicas de cultivo in vitro con los ensayos de campo, con el fin de lograr resultados consistentes.

- En la obtención de plantas transgénicas con alta tolerancia a aluminio, la transducción de material genético que induzca en ellas una mayor secreción de ácidos orgánicos, es hasta el momento uno de los objetivos, pues esta característica le conferiría a los vegetales una mayor posibilidad de detoxificación del aluminio.

\section{REFERENCIAS BIBLIOGRÁFICAS}

Aponte de L. M.E.; G. Valencia A. 1983. Toxicidad de aluminio en plantas de café. Cenicafe 34(3), 61-97.

Baier, A.C.; D.J. Somers y P. Gustafson. 1996. Aluminum tolerance in triticale, wheat and rye. pp. 437-444. En: Guedes-Pinto, P.; N. Darvey y V.P. Carnide (eds.). Triticale today and tomorrow. Kluwer Acad. Publ., Dordrecht.

Bartlett, R.J. y D.C. Riego. 1972. Effect of chelation on the toxicity of aluminum. Plant Soil 37, 419-423.

Berthelin, J.; G. Belgy; D. Boymond y C. Leyval. 1993. Microbial biosorption and ion accumulation of metals in field conditions in heavy metal polluted soils and 
around uranium mining wastes. En: Proc. FEMS Symposium Metals-Microorganisms: Relationships and Applications. Metz, Francia.

Blancaflor, E.B.; D.L. Jones y S. Gilroy. 1998. Alterations in the cytoskeleton accompany aluminum-induced growth inhibition and morphological changes in primary roots of maize. Plant Physiol. 118(1), 159-172.

Calbo, A.C. y J. Cambraia. 1980. Efeito do aluminio sobre a composição mineral de dois cultivares de sorgo (Sorghum bicolor L. Moench). Revista Ceres 37 , 379-384.

Cambraia, J.; M.A. da Silva; M.A.O. Cano; R. Sant'ana. 1991. Método simples para avaliação de cultivares de sorgo quanto a tolerancia ao alumínio. Rev. Bras. Fisiol. Veg. 3(2), 87-95.

Casierra-Posada, F. 2001. Fundamentos fisiológicos, bioquímicos y anatómicos del estrés por aluminio en vegetales. Revista Comalfi 28(2), 8-19.

Casierra-Posada, F. 2002. Alteraciones inducidas por aluminio en el citoesqueleto de las plantas. Revista Comalfi 29(2), 23-30.

Casierra-Posada, F.; J.F. Cárdenas-Hernández. 2007. Influencia del aluminio sobre el crecimiento de la raíz en coliflor (Brassica oleracea L., var. Botrytis, Hib. 'Nevada F1'). Revista UDCA Actualidad \& Divulgación Científica 10(1), 149-157.

Caniato, F.F.; C.T. Guimarães; R.E. Schaffert; V.M.C. Alves; L.V. Kochian; A. Borém; P.E. Klein y J.V. Magalhaes. 2007. Genetic diversity for aluminum tolerance in sorghum. Theor. Appl. Genet. 114(5), 863-876.

Collet, L.; C. de Leon y W.J. Horst, 2000. Screening maize for adaptation to aluminium-toxic soils of Colombia. Tagungsband 3.3. Deutscher Tropentag 2000. Hohenheim, Alemania.

Colpaert, J.V. y J.A. van Assche. 1992. Zinc toxicity in ectomycorrhizal Pinus silvestris. Plant Soil 143, 201-211.

Cumming, J.R. y L.H. Weinstein. 1990. Aluminum-mycorrizal interactions in the physiology of pitch pine seedlings. Plant Soil 125, 7-18.

De la Fuente, J.M.; V. Ramírez-Rodríguez; L. Cabrera-Ponce y L. Herrera-Estrella. 1997. Aluminum tolerance in transgenic plants by alteration of citrate synthesis. Science 276, 1566-1568.

De Lima, M. y L. Copeland. 1994. Changes in the intrastructure of the root tip of wheat following exposure to aluminum. Aust. J. Plant Physiol. 21, 85-94.

Duong, T.P.; C.N. Diep. 1986. An inexpensive cultural system using ash for cultivation of soybean (Glycine max (L.) Merrill) on acid clay soils. Plant Soil 96, 225-237.
Ezaki, B.; M. Sivaguru; R. Gardner y H. Matsumoto. 2000. Saccharomyces cerevisiae as a model system for the study of aluminum (Al) resistance mechanism in plants. Recent. Res. Dev. Microbiology 4, 67-75.

Foy, C.D. 1988. Plant adaptation to acid soil, aluminiumtoxic soils. Commun. Soil Sci. PlantAnal. 19, 959-987.

Ginting, S.; B.B. Johnson y S. Wilkens. 1998. Alleviation of aluminum phytotoxicity on soybean growth by organic anions in nutrient solutions. Aust. J. Plant Physiol. 25, 901-908.

Guerrero, R. 1991. La acidez del suelo - Su naturaleza, sus implicaciones y su manejo. pp. 141-163. En: Silva M. (ed.). Fundamentos para la interpretación de análisis de suelos, plantas y aguas para riego. Sociedad Colombiana de la Ciencia de Suelo. Montoya \& Araújo Ltda. Impresiones, Bogotá.

Hue, N.V. y I. Amien. 1989. Aluminium detoxification with green manure. Commun. Soil Sci. Plant Anal. 20, 1499-1511.

Hue, N.V.; G.R. Craddock; F. Adams. 1986. Effect of organic acids on aluminum toxicity in subsoils. Soil Sci. Soc. Amer. J. 50, 28-34.

Instituto Colombiano Agropecuario. 1992. Fertilización en diversos cultivos - Quinta aproximación. Manual de asistencia técnica No. 25. Produmedios. Bogotá. 64 p.

Kerven, G.L.; D.G. Edwards; C.J. Asher y S. Kokot. 1989. Aluminium determination in soil solution . II. Shortterm colorimetric procedures for the measurement of inorganic aluminium in the presence of organic acid ligands. Aust. J. Soil Res. 27, 91-102.

Kloke, A. 1978. Der Einfluß von Schadgasen und Schadstoffen auf die Vegetationsdecke. En: Olschowy, G. editor. Natur- und Umweltschutz in der BRD. Paul Parey, Berlin y Hamburgo.

Kochian, L.V. 1995. Cellular mechanisms of aluminum toxicity and resistance in plants. Annu. Rev. Plan. Physiol. Mol. Biol. 46, 237-260.

Larsen, P.B.; L.M. Stenzler; C.Y. Tai; J. Degenhardt; S.H. Howell y L.V. Kochian. 1997. Molecular and physiological analysis of Arabidopsis mutants exhibiting altered sensitivities to aluminum. Plant Soil 192, 3-7.

Ma, J.F. 2000. Role of organic acids in detoxification of aluminum in higher plants. Plant Cell Physiol. 41(4), 383-390.

Marschner, H. 1995. Mineral nutrition of higher plants. Segunda edición. Academic Press, Londres. pp. 606-613.

Marschner, P.; A. Klam; G. Jentschke y D.L. Godbold. 1999. Aluminium and lead tolerance in ectomicorrhizal fungi. J. Plant Nutr. Soil Sci. 162, 281-286. 
Matzner, E. y J. Prenzel. 1992. Acid deposition in german solling area - Effects on soil solution chemistry and Al movilization. Water, Air, Soil Pollut. 61, 221-234.

Méndez Diniz, V.P. y M.E. Ribeiro Calbo. 1990. Efeito da aplicação foliar de fósforo sobre a toxidez de alumínio em plantas de tomate. Rev. Bras. Fisiol. Vegetal 2(2), 57-61.

Oettler, G.; R. Geiger; S. Wiethölter; C.S. Gaus; C.U. Hesemann y W.J. Horst. 1997. Methodische und genetische Untersuchungen zur Aluminiumtoleranz von Triticale. Vortr. Pflanzenzüchtung 38, 41-56.

Ownby, J.D. y H.R. Popham. 1989. Citrate reverses the inhibition of wheat root growth caused by aluminum. J. Plant Physiol. 135, 588-591.

Pintro, J.; J. Barloy y P. Fallavier. 1995. Aluminium toxicity in corn plants cultivated in a low ionic strength nutrient solution. I. Discrimination of two corn cultivars. Rev. Bras. Fisiol. Veg. 7(2), 121-128.

Qiu-Ying T.; S. Dong-Hua; Z. Min-Guiy Z. Wen-Hao. 2007. Inhibition of nitric oxide synthase (NOS) underlies aluminum-induced inhibition of root elongation in Hibiscus moscheutos. New Phytol. 174, 322-331.

Ryan, P.R.; J.E. Shaff y L.V. Kochian. 1992. Aluminum toxicity in roots: Correlation among ionic currents, ion fluxes, and root elongation in aluminum-sensitive and aluminum-tolerant wheat cultivars. Plant Physiol. 99, 1193-1200.

Scheffer, F. y P. Schachtschabel. 1995. Lehrbuch der Bodenkunde. Ferdinand Enke, Stuttgart. pp. 313-316.
Sivaguru, M.; H. Matsumoto y W. Horst. 2000. Control of the response to aluminum stresss. En: Nick, P. (ed.). Plant microtubules: Potential for microbiology. Springer, Heidelberg. pp. 103-120.

Suthipradit, S.; D.G. Edwards y C.J. Asher. 1990. Effects of aluminium on tap-root elongation of soybean (Glycine max), cowpea (Vigna unguiculata) and greengram (Vigna radiata) grown in the presence of organic acids. Plant Soil 124, 233-237.

Tobin, J.M.; D.G. Cooper y R.J. Neufeld. 1990. Investigations of the mechanism of metal uptake by Rhyzopus arrhizus biomass. Enzime Microb. Technol. 12, 591-595.

Wagatsuma, T.; M. Kaneko y Y. Hayasaka. 1987. Destruction process of plant roots cells by aluminum. Soil Sci. Plant Nutr. 33, 16-175.

Wallnöfer, P.R. y G. Engelhardt. 1995. Schadstoffe aus dem Boden. 118-140 p. En: Hock, B. y E. Elstner (eds.). Schadwirkungen auf Pflanzen. Académica Spektrum, Berlin.

Wissemeier, A.H.; G. Hahn y H. Marschner. 1998. Callose in roots of norway spruce (Picea abies (L.) Karst) is a senditive parameter for aluminium supply at a forest site (Höglwald). Plant Soil 199, 53-57.

Wright, R.J. 1989. Soilaluminum toxicity and plant growth. Commun. Soil Sci. Plant Anal. 20, 1479-1497.

Zapata H. R.D. 2004. La química de la acidez del suelo. Disponible en: http://www.unalmed.edu.co/\%7Erdzapata/AcidezdelSuelo.zip; consulta: 12 de mayo de 2007. 